\title{
Human endogenous retroviruses and chosen disease parameters in morphea
}

\author{
Michał J. Kowalczyk ${ }^{1}$, Aleksandra Dańczak-Pazdrowska², Beata Szramka-Pawlak², Ryszard Żaba', \\ Agnieszka Osmola-Mańkowska ${ }^{4}$, Wojciech Silny $^{3}$
}

'Department of Dermatology and Venereology, Poznan University of Medical Sciences, Poznan, Poland

'Unit of Noninvasive Diagnostics of Skin Diseases, Department of Dermatology, Poznan University of Medical Sciences, Poznan, Poland ${ }^{3}$ Department of Dermatology, Poznan University of Medical Sciences, Poznan, Poland

${ }^{4}$ Psoriasis and Novel Therapies Unit, Department of Dermatology, Poznan University of Medical Sciences, Poznan, Poland

Adv Dermatol Allergol 2017; XXXIV (1): 47-51

DOI: https://doi.org/10.5114/ada.2017.65621

\begin{abstract}
Introduction: Morphea (localized scleroderma) is a relatively rare disease characterized by excessive skin fibrosis. Human endogenous retroviruses (HERV) are largely distributed within the human genome with hundreds of thousands of elements. The HERV have been widely studied in autoimmune disorders, yet hardly ever assessed in diseases with a good prognosis such as morphea.

Aim: In this study we focus on the possible relations between the expression of chosen HERV and factors influencing the pathomechanism of the disease, such as age, sex, titres of anti-nuclear antibodies, as well as duration, activity, and severity of the disease (LoSSI index).

Material and methods: Real-time polymerase chain reaction (PCR) targeting six HERV sequences of interest were performed on samples derived from peripheral blood mononuclear cells (PBMC) and skin biopsies.

Results: In PBMC we found a statistically significant negative correlation between HERV-W env expression and LoSSI index $(p=0.01)$. Additionally, HERV-W env was downregulated in patients with the active form of morphea. In all other cases we found no correlation whatsoever nor statistically significant differences below the $p=0.05$ threshold. Conclusions: Morphea seems to be an autoimmune disease where the impact of HERV is not so apparent. It seems that probing many patients for the expression of just a few sequences is not as effective as previously expected. For initial studies of HERV in other diseases we recommend high throughput techniques such as HERV-dedicated DNA microarrays or massive parallel sequencing.
\end{abstract}

Key words: human endogenous retroviruses, real-time polymerase chain reaction, autoimmunity, localized scleroderma, skin.

\section{Introduction}

Morphea or localized scleroderma (LS) is believed to be a skin-limited disease characterized by excessive skin fibrosis, in contrast to systemic sclerosis, which is additionally characterized by an internal organ involvement. Notwithstanding the two disorders sharing fibrosis, different molecular mechanisms might be involved. Morphea can be mimicked by other diseases including lichen sclerosus, sclerodermiform graft versus host disease, post-radiation dermatitis, progerias, drug-related sclerodermoid reactions and others [1-5].

Human Endogenous Retroviruses (HERV) are remnants of ancient retroviral infections, many of which have been preserved in genomes for millions of years. They have retained their original gene arrangement, although the accumulated mutations prevent them from forming infectious viral particles. Nonetheless, many of them generate mRNA and proteins that can directly influence cellular environment or indirectly exert changes by their own localization nearby or within other genes. Other possible mechanisms of interaction have also been reported in the literature [6-8].

\section{Aim}

In this study we focus on the possible relations between the expression of chosen HERV sequences and factors influencing the pathomechanism of the disease,

Address for correspondence: Michał J. Kowalczyk, Department of Dermatology and Venereology, 49 Przybyszewskiego St, 60-355 Poznan, Poland, phone: +61 531224 221, e-mail: michal.kowalczyk@test-dna.net Received: 9.11.2015, accepted: 10.02.2016. 
such as age, sex, anti-nuclear antibodies (ANA) titres, duration, activity, and severity of the disease.

The study involved expression assessment in peripheral blood mononuclear cells (PBMC) and skin biopsies. Six HERV sequences of particular interest that had been previously examined in other autoimmune disorders were chosen for this study.

\section{Material and methods}

\section{Groups of study}

Whole blood samples were collected from 41 patients with morphea (22 women, 19 men, mean age: 44.1, $\mathrm{SD}=17.5$ years) while skin biopsies from morphea lesions were collected from 36 patients (17 women, 19 men, mean age: $45.1 \pm 16.0$ years). The diagnosis was confirmed by histological examination. Patients were not under treatment at the time of sample collection. The exclusion criteria involved the presence of other chronic diseases, notably autoimmune disorders. Patients involved in this study had not been exposed to UV radiation for the period of at least a month prior to sample collection.

The study was approved by the Bioethics Committee of the Poznan University of Medical Sciences and

Table 1. Sequences of primers used in real-time PCR

\begin{tabular}{|c|c|c|}
\hline $\begin{array}{l}\text { Primer } \\
\text { name }\end{array}$ & Sequence $\left(5^{\prime}-3^{\prime}\right)$ & $\begin{array}{l}\text { Accession/ } \\
\text { location }\end{array}$ \\
\hline $\begin{array}{l}\text { HERV-E } \\
\text { pol F } \\
\text { HERV-E } \\
\text { pol R }\end{array}$ & $\begin{array}{l}\text { GTC ATT TGT ATT CTA CCG GAG } \\
\text { AAT ACT GCA AAG TTT GGG AC }\end{array}$ & $\begin{array}{l}\text { Retrosearch: } \\
\text { Orf ID: } 8435 \\
\text { HERV ID: } 1109 \\
\text { Chr. Xq21.32 }\end{array}$ \\
\hline $\begin{array}{l}\text { HERV-H } \\
\text { (env62) F } \\
\text { HERV-H } \\
\text { (env62) R }\end{array}$ & $\begin{array}{l}\text { TAT GTC ATC CTC TAC CTC TCC C } \\
\text { CCA GCA GTT GTT CAC TAA GGA }\end{array}$ & $\begin{array}{l}\text { Retrosearch: } \\
\text { Orf ID: } 82113 \\
\text { HERV ID: } 10816 \\
\text { Chr. 2q24.3 }\end{array}$ \\
\hline $\begin{array}{l}\text { HERV-K } \\
\text { env F } \\
\text { HERV-K } \\
\text { env R }\end{array}$ & $\begin{array}{c}\text { CAC TTG GGT TAA GAC CAT } \\
\text { TGG A } \\
\text { GGA GCT GTT GAG TAC ACC TG }\end{array}$ & $\begin{array}{l}\text { Retrosearch: } \\
\text { Orf ID: } 204173 \\
\text { HERV ID: } 29013 \\
\text { Chr. } 8 \text { p23.1 }\end{array}$ \\
\hline $\begin{array}{l}\text { HERV-K10 } \\
\text { gag F } \\
\text { HERV-K10 } \\
\text { gag R }\end{array}$ & $\begin{array}{l}\text { GTA ATG GCT CAG TCA ACG CA } \\
\text { GCC CCA TTA ATT CTG GAC CT }\end{array}$ & $\begin{array}{l}\text { GenBank: } \\
\text { M14123 }\end{array}$ \\
\hline $\begin{array}{l}\text { HERV-R } \\
\text { pol-env F } \\
\text { HERV-R } \\
\text { pol-env R }\end{array}$ & $\begin{array}{l}\text { GGG CCA ATT ATG CTT ACC AA } \\
\text { ATG GGC TGA TCT GGC TCT AA }\end{array}$ & $\begin{array}{l}\text { GenBank: } \\
\text { M12140 } \\
\text { Chr. 7q11.2 }\end{array}$ \\
\hline $\begin{array}{l}\text { HERV-W } \\
\text { env F } \\
\text { HERV-W } \\
\text { env R }\end{array}$ & $\begin{array}{l}\text { TCA TAT CTA AGC CCC GCA AC } \\
\text { GAG GTT GTG ATA CCG CCA AT }\end{array}$ & $\begin{array}{l}\text { GenBank: } \\
\text { AF072506 }\end{array}$ \\
\hline $\begin{array}{l}\text { GAPDH F } \\
\text { GAPDH R }\end{array}$ & $\begin{array}{c}\text { CTG CAC CAC CAA CTG CTT AG } \\
\text { TTC TGG GTG GCA GTG ATG }\end{array}$ & $\begin{array}{c}\text { Ensembl: } \\
\text { ENST00000229239 }\end{array}$ \\
\hline
\end{tabular}

performed according to the Declaration of Helsinki. All patients gave written consent.

\section{Peripheral blood mononuclear cells (PBMC) and plasma}

EDTA-collected blood was separated in Ficoll gradient $\left(1.077 \mathrm{~g} / \mathrm{cm}^{3}\right)$. Until examined, the aliquoted plasma was kept at the temperature of $-80^{\circ} \mathrm{C}$. The PBMC were washed in phosphate buffered saline (PBS) and disintegrated in TriPure reagent (Roche Applied Science). RNA was isolated with the use of a modified Chomczynski and Sacchi protocol [9]. 1 ug of each RNA sample was DNased to minimize genomic DNA contamination (Ambion, USA). Reverse transcription (RT) was performed with the use the Transcriptor First Strand cDNA Synthesis Kit (Roche Applied Science) with random hexamer primers. cDNA was stored in aliquots at $-80^{\circ} \mathrm{C}$ prior to real-time polymerase chain reaction (PCR). Each RNA sample was assessed through a PCR no-RT negative reaction.

\section{Skin biopsies}

Skin biopsies of morphea patients were collected from the centre of pathological areas under local anaesthetic. Shortly after collection, skin samples were frozen in liquid nitrogen and stored at the temperature of $-80^{\circ} \mathrm{C}$. Samples were disintegrated by rotor-stator homogenization carried out on ice in TriPure reagent. After removal of debris by centrifugation, RNA was isolated with the use of a modified Chomczynski and Sacchi protocol, followed by genomic DNA removal and cDNA synthesis [9]. The remaining steps were the same as in the case of PBMC.

\section{Real-time polymerase chain reaction (PCR)}

CDNA samples were amplified by real-time PCR in a LightCycler 2.0 thermocycler using LightCycler FastStart DNA Master SYBR Green I kit (Roche Diagnostics $\mathrm{GmbH}$, Germany) with additional uracil-n-glycosylase (Fermentas, Canada) for carry-over prevention. Standard curves were prepared with the use of amplicon samples of known concentrations. The results of HERV expression were normalized to the level of glyceraldehyde 3-phosphate dehydrogenase (GAPDH) transcription. The choice of this particular gene as a reference had been supported in the literature [10]. Sequences of primers for HERV-K10 gag, HERV-R pol-env, HERV-W env, and GAPDH were imported from our previous studies [11]. The sequences and accession data are provided in Table 1.

\section{Evaluation of anti-nuclear antibodies (ANA)}

The ANA were assessed by indirect immunofluorescence on a mosaic of substrates (neoplastic cell line of laryngeal carcinoma, HEp-2/monkey hepatocytes) with the use of commercially available kits (Euroimmun, Germany), according to the manufacturer's instructions. 
Table 2. Statistical analysis results for PBMC. Spearman's ranked correlation coefficient with respective $p$-values are presented for ANA titres, disease duration, LoSSI and age

\begin{tabular}{|c|c|c|c|c|c|c|c|}
\hline HERV sequence & & E pol & W env & $\mathrm{R}$ pol-env & H env 62 & K env & K10 gag \\
\hline \multirow[t]{2}{*}{ ANA titre } & $r_{s}$ & -0.15 & -0.17 & -0.10 & -0.11 & 0.03 & 0.04 \\
\hline & $P$-value & 0.33 & 0.28 & 0.52 & 0.50 & 0.87 & 0.82 \\
\hline \multirow[t]{2}{*}{ Disease duration } & $r_{s}$ & -0.18 & -0.16 & -0.04 & -0.02 & 0.00 & 0.00 \\
\hline & $P$-value & 0.26 & 0.32 & 0.81 & 0.91 & 0.98 & 0.98 \\
\hline \multirow[t]{2}{*}{ LoSSI } & $r_{s}$ & -0.22 & -0.38 & -0.24 & -0.08 & 0.04 & 0.15 \\
\hline & $P$-value & 0.18 & $0.01^{*}$ & 0.13 & 0.61 & 0.78 & 0.34 \\
\hline \multirow[t]{2}{*}{ Age } & $r_{s}$ & -0.23 & 0.13 & 0.08 & 0.26 & -0.18 & 0.07 \\
\hline & $P$-value & 0.15 & 0.41 & 0.63 & 0.10 & 0.27 & 0.66 \\
\hline Sex & $P$-value & 0.31 & 0.14 & 0.87 & 0.24 & 0.61 & 0.40 \\
\hline Disease activity & $P$-value & 0.13 & $0.04^{\star}$ & 0.45 & 0.65 & 0.43 & 0.79 \\
\hline
\end{tabular}

P-values of $U$ tests are presented for sex and disease activity. *Statistically significant instances are marked.

\section{Evaluation of activity of the disease}

An appearance of a new lesion, spread of a previously existing one or a presence of erythematous margins within the recent 6 months qualified a patient to the group with an active process $[12,13]$.

\section{Lesion assessment}

Morphea lesions were evaluated employing the Localized Scleroderma Severity Index (LoSSI) suggested by Arkachaisri and Pino. The maximum possible score was 168 [14].

\section{Statistical analysis}

Normality of collected data sets was assessed with the Shapiro-Wilk test, while significant outliers were detected with the Grubbs' test (data not published). Since the vast majority of data was not normally distributed, and there were two independent samples, the WilcoxonMann-Whitney $U$ test was utilized for the assessment of the differences between sexes and disease activity. In the study of age, ANA titres, duration and severity of the disease, the Spearman's ranked correlation coefficient was calculated. Statistical analysis as well as the graphs were computed with the use of Past v.2.03 software.

\section{Results}

Prior to real-time analyses, optimization was carried out separately for each type of HERV sequence. A melting curve analysis was performed for all reactions and the results were compared to the standard melting temperatures found during the optimization process.

Randomly chosen reaction batches were duplicated for pipetting error analysis. In such cases, mean values of the two reactions were used in further statistical analysis.

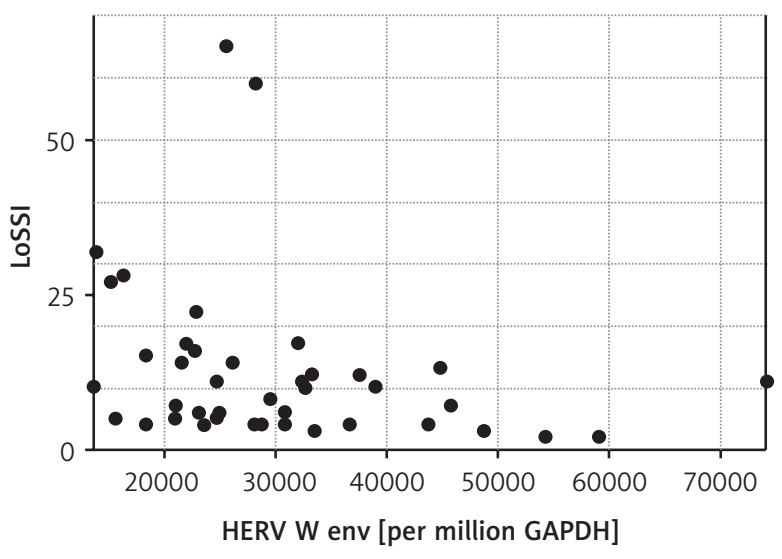

Figure 1. Correlation between the expression of HERV $W$ env in PBMC and Localized Scleroderma Severity Index (LoSSI). $r_{s}=-0.38, p=0.01$. HERV $W$ env expression level is expressed in copies of HERV W env per million copies of the GAPDH reference gene

Results of the statistical analyses concerning PBMC are presented in Table 2. We found no statistically significant correlations between the expression of all HERV sequences and ANA titre, disease duration and age. The expression of HERV W env sequence was the only case found to correlate with LoSSI (Figure 1). There were no statistically significant differences between sexes in the expression of the aforementioned sequences. The only statistically significant difference in the case of disease activity was the instance of HERV W env.

Results of the statistical analyses concerning skin biopsies are presented in Table 3. Here we found no statistically significant correlations between the expression of all HERV sequences and all tested parameters. HERV H env 62 positively correlated with age with the $p$-value close to statistical significance. There were no statistically significant differences between sexes and 
Table 3. Statistical analysis results for skin biopsies. Spearman's ranked correlation coefficient with respective $p$-values are presented for ANA titres, disease duration, LoSSI and age

\begin{tabular}{lcccccccc}
\hline HERV sequence & & E pol & W env & R pol-env & H env 62 & K env & K10 gag \\
\hline ANA titre & $r_{s}$ & -0.25 & -0.21 & -0.16 & -0.03 & -0.14 & 0.08 \\
\cline { 2 - 8 } & $P$-value & 0.13 & 0.22 & 0.35 & 0.87 & 0.43 & 0.63 \\
\hline \multirow{2}{*}{ Disease duration } & $r_{s}$ & 0.10 & 0.20 & 0.07 & 0.03 & 0.10 & -0.04 \\
\cline { 2 - 9 } & $P$-value & 0.55 & 0.23 & 0.70 & 0.85 & 0.57 & 0.82 \\
\cline { 2 - 9 } & $r_{s}$ & 0.07 & -0.15 & -0.08 & -0.01 & -0.05 & -0.10 \\
\hline AgSSI & $P$-value & 0.70 & 0.40 & 0.66 & 0.95 & 0.79 & 0.60 \\
\hline Sex & $r_{s}$ & 0.17 & 0.21 & 0.11 & 0.31 & 0.25 & 0.23 \\
\hline Disease activity & $P$-value & 0.33 & 0.23 & 0.52 & $0.06^{*}$ & 0.15 & 0.17 \\
\hline P-value & $P$-value & 0.82 & 0.27 & 0.90 & 0.21 & 0.50 & 0.55 \\
\hline
\end{tabular}

$P$-values of $U$ tests are presented for sex and disease activity. ${ }^{*} A n$ instance close to statistical significance is marked.

disease activity in the expression of the aforementioned sequences.

\section{Discussion}

In the vast majority of cases we have found no correlation whatsoever nor statistically significant differences, with the exception of HERV W. In PBMC we found a statistically significant negative correlation with respect to LoSSI. The more severe the disease, the smaller the expression of HERV W env. This phenomenon is also supported by a statistically significant difference in the expression of this sequence between patients with an active and patients with a non-active disease. The former show a decrease of transcription (data not shown). We compared these results to a healthy control group in a parallel study and were surprised to find out that in skin morphea patients did show a downregulation, which supports these data, but in PBMC there was an upregulation of HERV W env expression in morphea [15]. In other words, although in PBMC the expression of HERV $W$ env is statistically higher in morphea, patients with more severe symptoms tend to have their expression lowered. Perhaps such phenomenon could be explained if the expression of HERV W env somehow correlated with the duration of the disease, yet it did not. The cause of such result is unknown.

As far as the skin is concerned, one cannot overlook a positive correlation between age and HERV H env 62 expression, where the $p$ value equals 0.06 , which is slightly above the commonly assumed $5 \%$ threshold. The same sequence shows traces of correlation in PBMC, where the $p$-value equals 0.10 . A parallel study performed in healthy people shows no signs of such correlation between HERV H env 62 expression level and age either in PBMC $(n=36$, $\left.r_{\mathrm{s}}<0.01, p=0.98\right)$ or in the $\operatorname{skin}\left(n=13, r_{\mathrm{s}}=0.23, p=0.46\right)$.

Our previous study shows a strong downregulation of HERV H env 62 expression in the skin of morphea patients but not in PBMC [15]. This could lead to a conclu- sion that the younger the patient, the bigger the ability to decrease the expression of HERV H env 62 in the skin. The literature study shows no apparent reason why the expression of this particular sequence but no other show such correlation. Although mysterious, this correlation most probably has little scientific value, being quite weak and just over the statistical significance level.

Studies on Human Endogenous Retroviruses are flourishing. The number of viral sequences to be studied is vast and the popularity of the subject among scientists has been steadily increasing. The studies are no longer limited to the mainstream of HERV research areas such as oncogenesis, mammalian embryogenesis, or genomics and are inexorably expanding onto other fields of science [16-18]. Although the importance of these studies cannot be underestimated, the influence of endogenous viruses on human disorders does have its limits. Morphea seems to be an autoimmune disease where the impact of HERV is not so apparent. Although there are differences in the levels of transcription of certain HERV sequences between morphea and healthy people [15], these values tell us little about the disease pathogenesis, as hardly any cause or effect of such changes is clear.

It seems that probing many patients for the expression of just a few sequences is not as effective as previously expected. For future initial studies of HERV in other diseases we recommend high throughput techniques such as HERV-dedicated DNA microarrays or massive parallel sequencing which are already commercially available.

\section{Acknowledgments}

Funded by the State Committee for Scientific Research under grants no. KBN N402 371338 and no. KBN N402 040 31/1276.

\section{Conflict of interest}

The authors declare no conflict of interest. 


\section{References}

1. Kreuter A, Krieg T, Worm M, et al. Diagnosis and therapy of localized scleroderma. J Detsch Dermatol Ges 2009; 7 (Suppl 6): S1-14.

2. Reich A, Heisig M, Baran E. Werner syndrome - a premature aging syndrome that can mimic scleroderma. Postep Dermatol Alergol 2010; 27: 490-4.

3. Nedoszytko B. Genetically determined syndromes associated with premature aging. Postep Dermatol Alergol 2010; 27: 282-90.

4. Szymanek M, Chodorowska G, Kowal M, et al. Serum soluble Fas levels in patients with systemic sclerosis. Postep Dermatol Alergol 2010; 27: 406-14.

5. Peterson LS, Nelson AM, Su WP. Classification of morphea (localized scleroderma). Mayo Clin Proc 1995; 70: 1068-76.

6. Urnovitz HB, Murphy WH. Human endogenous retroviruses: nature, occurrence, and clinical implications in human disease. Clin Microbiol Rev 1996; 9: 72-99.

7. Zwolinska K. Retroviruses-derived sequences in the human genome. Human endogenous retroviruses (HERVs). Postep Hig Med Dosw 2006; 60: 637-52.

8. Larek-Rąpała A, Żaba R, Kowalczyk MJ, et al. Herpes simplex virus infection as a possible modulator of autoimmune diseases facilitated by human endogenous retroviruses. Postep Dermatol Alergol 2011; 28: 313-6.

9. Chomczynski P, Sacchi N. Single-step method of RNA isolation by acid guanidinium thiocyanate-phenolchloroform extraction. Anal Biochem 1987; 162: 156-9.

10. Bonnet-Duquennoy M, Abaibou H, Tailhardat M, et al. Study of housekeeping gene expression in human keratinocytes using OLISATM, a long-oligonucleotide microarray and q RTPCR. Eur J Dermatol 2006; 16: 136-40.

11. Namyst J, Osmola A, Prokop J. Human endogenous retroviruses sequences expression in patients with progressive systemic sclerosis and different clinical forms of psoriasis. Postep Dermatol Alergol 2005; 22: 99-10.

12. Marzano AV, Menni S, Parodi A, et al. Localized scleroderma in adults and children. Clinical and laboratory investigations on 239 cases. Eur J Dermatol 2003; 13: 171-6.

13. Uziel Y, Feldman BM, Krafchik BR, et al. Increased serum levels of TGFbeta1 in children with localized scleroderma. Pediatr Rheumatol Online J 2007; 5: 22.

14. Arkachaisri T, Pino S. Localized scleroderma severity index and global assessment: a pilot study of outcome instruments. J Rheumatol 2008; 35: 650-7.

15. Kowalczyk MJ, Dańczak-Pazdrowska A, Szramka-Pawlak B, et al. Expression of chosen human endogenous retroviral sequences in skin and PBMCs in morphea. Arch Med Sci 2012; 8: 819-25.

16. Balestrieri E, Pitzianti M, Matteucci C, et al. Human endogenous retroviruses and ADHD. World J Biol Psychiatry 2014; 15: 499-504.

17. Mameli G, Madeddu G, Mei A, et al. Activation of MSRV-type endogenous retroviruses during infectious mononucleosis and Epstein-Barr virus latency: the missing link with multiple sclerosis? PLoS One 2013; 8: e78474.

18. Assinger A, Yaiw KC, Göttesdorfer I, et al. Human cytomegalovirus (HCMV) induces human endogenous retrovirus (HERV) transcription. Retrovirology 2013; 10: 132. 